\title{
3D printing technique for guiding complicated cardiovascular surgery
}

\author{
Nan Chen ${ }^{1,2 *}$, Kai Zhu ${ }^{1,2 *}$, Chunsheng Wang ${ }^{1,2}$, Xiaoning Sun ${ }^{1,2}$ \\ ${ }^{1}$ Department of Cardiac Surgery, Zhongshan Hospital, Fudan University, Shanghai 200032, China; ${ }^{2}$ Shanghai Institute of Cardiovascular Disease, \\ Shanghai 200032, China \\ *These authors contributed equally to this work. \\ Correspondence to: Xiaoning Sun, MD, PhD. Department of Cardiac Surgery, Zhongshan Hospital, Fudan University, and Shanghai Institute of \\ Cardiovascular Disease, Shanghai 200032, China. Email: sun.xiaoning@zs-hospital.sh.cn. \\ Provenance: This is an invited article commissioned by the Section Editor Kai Zhu (Department of Cardiac Surgery, Zhongshan Hospital Fudan \\ University, Shanghai, China). \\ Response to: Ong CS, Hibino N. The use of 3D printing in cardiac surgery. J Thorac Dis 2017;9:2301-2. \\ Rosu C, Demers P. Three-dimensional printing in cardiovascular surgery: logical next step after three-dimensional imaging. J Thorac Dis \\ 2017;9:2720-2.
}

Submitted Aug 17, 2017. Accepted for publication Aug 20, 2017.

doi: $10.21037 /$ jtd.2017.08.157

View this article at: http://dx.doi.org/10.21037/jtd.2017.08.157

The impressive guidance of three-dimensional (3D) printed model in surgery for a patient with right-sided aortic arch and Kommerell's diverticulum, being presented in our former paper (1), leading to a sound outcome, has been highlighted as well as commented in the editorials which means a big honor for us. Kommerell' diverticulum has been established a rare abnormity in some researches $(2,3)$, even may be seen only once for some surgeons in their career, the medical management varies due to the rarity and complexity of this disease, including surgery, hybrid procedure, and conservative treatment with drugs. The surgical procedure of healing Kommerell's diverticulum is somehow unfamiliar to surgeons, to some extend may be difficult, especially associated with a right-sided aortic arch. The way we introduced in our paper (1) way be a good experience to go by for cardiac surgeons.

As mentioned by all physicians, the novel $3 \mathrm{D}$ reconstruction of cross-sectional imaging has been an advanced and ubiquitous technique in assisting diagnosis as well as planning and execution of medical management in many fields, such as orthopedic surgery (4), accounting for the biggest population, neurological surgery (5), plastic surgery (6), and cardiovascular surgery (7), to some degree making the therapy being of better efficiency, accuracy and precision. Compared with the digital reconstruction, the physical one, pointing to the printed model, carries a preferable vision and more excellent presence of the anatomy which can be graspable as well as obviating the mental manipulation required in analyzing digital objects on a screen after post-processing in various software and printing machines with the computed tomographic angiography (CTA) or magnetic resonance imaging (MRI) data. As described in Treasure's paper, the word 'graspable', meaning an object which surgeons can be grasp and manipulate in hands even simulate surgery on it, makes a dramatic point. The direct touch and advanced vision provided by the model promises a better understanding and more detailed preoperative planning, the same as 'more information means better outcome' wrote by Sundararaghavan (8). It is currently in congenital heart disease where the $3 \mathrm{D}$ printing has been most employed for cardiac surgeons, as well has been extended to acquired heart disease. The congenital heart disease is often of great complexity and the hemodynamics are mutable. Utilization of $3 \mathrm{D}$ models provide an excellent view of the complexity to the surgeons as well as the simulation of operation before procedure.

With all its numerous advantages and uses of the emerging technique being demonstrated and proofed above, being accompanied by some limitations (7), 3D printing is still some years away from being ubiquitous as CTA reconstruction or MRI in cardiovascular medicine so far. What should be noted is that the rapid prototyping (RP) industry came 
to public more than 3 decades ago, but it was just the last decade that RP was used in medical applications and named as ' $3 \mathrm{D}$ printing'. The gap in the initiating moment tells the lack of communication and teamwork between physicians and engineers. Tom Treasure and his team, mainly with John Pepper, have showed us a successful collaboration which can overcome the 'gap' limitation to follow up. Secondly, the 'expensive' issue which has been continuously debated ever since the utilization of $3 \mathrm{D}$ printing in medical applications dose restrict the prevalence of this novel technology. The generation of a $3 \mathrm{D}$ printed model is such a complex workflow, involving the acquisition of imaging data, the postprocessing, the digital reconstruction, the printing procedure and some other related to it, not only expensive but also time-costing, that can't be afforded by some situations. Better would it be if the industry program was simplified and the printing achieved in-hospital. Thirdly, the availability of materials which can reproduce cardiovascular structure holding the most of their characteristics is still limited. Structure delicate as valves and sub-valves, tiny as coronary artery, complex as myocardium, flexible as great vessels, are all difficult to be reproduced with all their properties being remained. Moreover, robust evidence and explicit researches demonstrating $3 \mathrm{D}$ printing can significantly improve outcome and patient care is still short-landed.

It is obvious that $3 \mathrm{D}$ printing will have an integral role in the multi-disciplinary and collaborative cardiovascular diagnosis and management strategies. The feasibility of this technique has been shown for a broad range of cardiovascular diseases. From perspectives of future progress, some issues should be highlighted, involving some of above and others. First of all, a big progress should be made in the model-generation industry because a simplified and cheaper one will be vital for the prevalence of the $3 \mathrm{D}$ print technique as a mainstream with an acceptable costs-outcomes ratio in the imaging division. Furthermore, large patient cohort prospective studies, which can lead to large-scale, randomized, clinical trials, focusing on the specific clinical role that $3 \mathrm{D}$ printing plays, the powerful evidence of $3 \mathrm{D}$ printing promoting clinical outcomes, and the firm demonstration of effectiveness are urgently required. Lastly, researches on bio-printing procedure involving the bioink materials, printing techniques, extracellular matrix (ECM), as well as scaffolds, need to put forward in cardiovascular surgery (9).

\section{Acknowledgements}

Funding: We thank for the funding sponsored by Shanghai
Pujiang Program (No. 14PJD008 and 17PJ1401500), National Natural Science Foundation of China (No. 81771971) "Chen Guang" Project Supported by Shanghai Municipal Education Commission and Shanghai Education Development Foundation (Grant No. 14CG06).

\section{Footnote}

Conflicts of Interest: The authors have no conflicts of interest to declare.

\section{References}

1. Chen N, Zhu K, Zhang H, et al. Three-dimensional printing guided precise surgery for right-sided aortic arch associated with Kommerell's diverticulum. J Thorac Dis 2017;9:1639-43.

2. Idrees J, Keshavamurthy S, Subramanian S, et al. Hybrid repair of Kommerell diverticulum. J Thorac Cardiovasc Surg 2014;147:973-6.

3. Cinà CS, Althani $\mathrm{H}$, Pasenau J, et al. Kommerell's diverticulum and right-sided aortic arch: a cohort study and review of the literature. J Vasc Surg 2004;39:131-9.

4. Huang TW, Hsu RW. Computer-assisted orthopedic surgery. Formosan Journal of Musculoskeletal Disorders 2016;7:1-7.

5. Randazzo M, Pisapia JM, Singh N, et al. 3D printing in neurosurgery: A systematic review. Surg Neurol Int 2016;7:S801-9.

6. Kamali P, Dean D, Skoracki R, et al. The Current Role of Three-Dimensional Printing in Plastic Surgery. Plast Reconstr Surg 2016;137:1045-55.

7. Giannopoulos AA, Mitsouras D, Yoo SJ, et al. Applications of 3D printing in cardiovascular diseases. Nat Rev Cardiol 2016;13:701-18.

8. Sundararaghavan S. Three-dimensional-printed cardiac prototypes in complex congenital cardiac defects: New technology with exciting possibilities. Ann Pediatr Cardiol 2017;10:114-6.

9. Duan B. State-of-the-Art Review of 3D Bioprinting for Cardiovascular Tissue Engineering. Ann Biomed Eng 2017;45:195-209.

Cite this article as: Chen N, Zhu K, Wang C, Sun X. 3D printing technique for guiding complicated cardiovascular surgery. J Thorac Dis 2017;9(10):E945-E946. doi: 10.21037/ jtd.2017.08.157 